\title{
Profile of Dermatophyte and Non Dermatophyte Fungi in Patients Suspected of Dermatophytosis
}

\author{
Gebreabiezgi Teklebirhan", Adane Bitew ${ }^{2, *}$ \\ ${ }^{1}$ Tikur Anbessa Hospital, College of Health Sciences, Addis Ababa University, Addis Ababa, Ethiopia \\ ${ }^{2}$ Department of Medical Laboratory Sciences, College of Health Sciences, Addis Ababa University, Addis Ababa, Ethiopia
}

Email address:

gebreab4@gmail.com(G. Teklebirhan), bitewadane@gmail.com (A. Bitew)

\section{To cite this article:}

Gebreabiezgi Teklebirhan, Adane Bitew. Profile of Dermatophyte and Non Dermatophyte Fungi in Patients Suspected of Dermatophytosis. American Journal of Life Sciences.Vol. 3, No. 5, 2015, pp. 352-357.doi: 10.11648/j.ajls.20150305.13

\begin{abstract}
Fungal infections of hair, nail and skin are common worldwide and continue to increase. The present study was undertaken to determine the prevalence of dermatophytosis, isolation rates and the profile of fungi associated with dermatophytosis. Samples were collected from 305 patients and a portion of each sample was examined microscopically and the remaining portion was cultured on to plates of Sabouraud's Dextrose Agar containing chloramphinical with and without cychloheximide. Fungal cultures were identified by studying macroscopic and microscopic characteristics of their colonies. Of 305 clinical samples, fungi were detected in 265 (86.9\%) samples by $\mathrm{KOH}$ and $224(73.4 \%)$ clinical samples were culture positive. Dermatophytes were the most common isolates accounting $130(58.0 \%)$ of the total isolates and this was followed by non- dermatophyte molds and yeasts that accounted 49 (21.9\%.) and $45(20.0 \%)$ respectively. T. violaceum was the dominant species accounting for 49 (37.7\%) dermatophyte isolates. Candida albicans was the dominant species accounting 30 (66.7\%) of the total yeast isolates. The genus Aspergillus was the most common non-dermatophyte molds consisting of 13 (26.5\%) species. Tinea unguium was the predominant clinical manifestation accounting $51.1 \%$ of the cases of which $119(76.3 \%)$ were from females and $37(23.7 \%)$ from males. T. violaceum was the most common pathogen in tinea unguium and tinea capitis, whereas T. mentagrophytes was the most common pathogen in tinea pedis and tinea manum. Seventy five percent of yeasts and $77.6 \%$ non dermatophyte molds were isolated from nails. Along with dermatophytes, non-dermatophyte fungi are also emerging as important causes of dermatophytosis. Both direct microscopy and culture are important tools for diagnosis of the fungal infections.
\end{abstract}

Keywords: Dermatophytosis, Non Dermtophyte Fungi, Trychophyton violaceum, Tinea unguium

\section{Introduction}

Superficial mycoses have been found in the last few decades to affect $20-25 \%$ of the world's population, making them one of the most frequent forms of infection [1]. Among superficial mycoses, dermatophytosis (dermatomycosis or ringworm infection) is the most common contagious infection all over the world. It is a fungal infection of the outermost layer of skin and its appendages such as hair and nails $[2,3]$. The mycosis is caused by forty different species of dermatophytes belonging to the genus Trichophyton, Microsporum and Epidermophyton [4, 5], and usually classified as different tinea depending up on the site of infection. Recently, association of non dermatophyte molds and yeasts with various forms of clinical manifestations of dermatophytosis has been documented by many researchers
[6-10]. Wide use of broad spectrum antibiotics, immunosuppressive drugs, invasive procedures and emergence of underlining diseases that suppress host immune system have been incriminated as major factors for their increase [11].

Dermatophyte and non- dermatophyte fungi implicated as a cause of dermatophytosis have been recorded all over the world but with variation in distribution, incidence, epidemiology, clinical manifestations and target hosts from one location to another. The heterogeneity in the distribution of dermatophytosis, their etiologic agents and the predominating clinical manifestation patterns in different parts of the world have been attributed to factors of geographic location, climate, overcrowding, health care, 
immigration, environmental hygiene culture and socioeconomic conditions $[1,12]$.

On the other hand, there are many reports that indicate the prevalence of dermatophytosis has significantly reduced in developed countries as opposed to developing ones and the reduction in the prevalence of dermatophytosis has been attributed to improved social, economic, health care and hygiene practice in the former [1, 13]. Ethiopia being a developing nation located in the tropic with wet humid climate appears to fall into the category of regions with high prevalence of dermatophytosis. Furthermore, Ethiopia as one of the developing countries, socioeconomic constraints and other common prevalent health issues have led to a low awareness of dermatophytosis by physicians and general population. Moreover, studies that investigated the prevalence of dermatophytosis and its etiologic agents in Ethiopia are few and most of them were carried out on a specific section of a population i.e. school children [14- 17], and these studies may not be a true representation of the overall disease pattern of the country. To this effect conducting research to understand the actual magnitude of dermtophytosis and the profile of its etiological agents among the general population at a various localities appears to be of the highest priority. Thus, this study was designed to determine the current prevalence and pattern of dermatophytosis in a tertiary hospital to which patients from all over the capital city and/ or outside the city are referred and thus can provide additional information on the trends of dermatophytosis. Findings from this study will provide up-to date information on dermatophytosis for evidence-based action aimed at reducing the morbidity of the infection.

\section{Materials and Methods}

\subsection{Sample Collection}

A total of 305 clinical samples were collected from patients visiting the dermatology department of Tikur Anbessa teaching Hospital, College of Health Sciences Addis Ababa University. The samples were collected from September 2014 to October 2015. Before collecting the samples the infected areas were cleaned with $70 \%(\mathrm{v} / \mathrm{v})$ ethanol. Then skin and nail samples were collected by scrapping of lesion with sterile blade and dull broken hairs from the margin of scalp lesion with forceps and transferred to sterile folded papers. Each of these papers was appropriately labeled with the age, sex, date of collection, code of a patient and location of infection and taken to the microbiology laboratory of the Department of Medical Laboratory Science, College of Health Sciences within the date of collection.

\subsection{Culture and Microscopic Examination}

A portion of each sample was mounted in a drop of an aqueous solution of $10 \%(\mathrm{w} / \mathrm{v})$ potassium hydroxide $(\mathrm{KOH})$ on a clean microscopic slide. After 5 minutes of mounting, the preparation was examined under low (X10) and high
(X40) power magnification for the presence of fungal elements. The remaining portion of each clinical sample was cultured irrespective of the negative or positive direct microscopic examination results on to duplicate plates of Sabouraud's Dextrose Agar (SDA) containing chloramphenicol with and without cychloheximide (Oxide, Basingstoke, England) which were prepared according to the manufacture's instruction. All inoculated plates were then incubated at inverted position for 4-6 weeks at $25^{\circ} \mathrm{C}$ and $37^{\circ} \mathrm{C}$ aerobically. Culture plates were examined twice a week for any fungal growth. Colonies suspected of dermatophytes and non-dermatophyte molds were sub-cultured into potato dextrose agar (Oxoid, Basingstoke, England) for the production of spores. Cultures of dermatophyte and non dermatophyte molds were identified by examining macroscopic and microscopic characteristics of their colony. Texture, rate of growth, topography and pigmentation of the front and the reverse side of the culture were employed for the macroscopic identification. Microscopic identification of mold isolates was performed by placing pieces of a colony from SDA and/or PDA to clean microscopic slide and staining with lactophenol cotton blue. After placing a cover slip, each preparation was observed microscopically. Urea agar (Oxoid, Basingstoke, England) was used in the differentiation of Trichophyton tonsurans, Trichophyton violaceum and Trichophyton rubrum. Yeasts were identified by employing conventional biochemical and assimilation test procedures [18] and using chromagar Candida culture medium (Becton Dickinson) as per the instruction of the manufacture. All ethical considerations and obligations were duly addressed and the study was conducted after the approval of the Department Research and Ethical Review Committee (DRERC) of the Department of Medical Laboratory Sciences, College of Health Sciences, Addis Ababa University. Informed written consent was obtained from participants before data collection. The respondent was given the right to refuse to take part in the study as well as to withdraw at any time during the study period. All the information obtained from the study subjects were coded to maintain confidentially. When the participants were found to be positive for fungal pathogen, they were informed by the hospitalclinician and received proper treatment.

\section{Result}

In the present study a total of 305 clinical samples were collected from suspected cases of dermatophytosis of which $97(31.8 \%)$ were from male and $208(68.2 \%)$ from female patients. The ages of study subjects ranged from 1 year to 80 years with a mean age of 26 years. The details regarding clinical manifestation and sex of study subjects were given in Table 1. Tinea unguium was the predominant clinical manifestation accounting $51.1 \%$ of the cases of which 119 $(76.3 \%)$ were from females and $37(23.7 \%)$ from males. This was followed by tinea corporis and tinea capitis accounting $20.0 \%$ and $10.8 \%$ of the cases respectively. 
Table 1. Frequency of clinical manifestations in relation to sex $(n=305)$.

\begin{tabular}{llll}
\hline Clinical & Sex & & \multirow{2}{*}{ Total } \\
\cline { 2 - 3 } Manifestation & Male & Female & \\
\hline Tinea capitis & 24 & 37 & $61(20 \%)$ \\
Tinea corporis & 7 & 26 & $33(10.8 \%)$ \\
Tinea cruris & 4 & 0 & $4(1.3 \%)$ \\
Tinea unguium & 37 & 119 & $156(51.1 \%)$ \\
Tjnea pedis & 6 & 9 & $15(4.9 \%)$ \\
Tinea faciei & 9 & 11 & $20(6.6 \%)$ \\
Tinea manum & 10 & 6 & $16(5.2 \%)$ \\
Total & $97(31.8 \%)$ & $208(68.2 \%)$ & $305(100.0 \%)$ \\
\hline
\end{tabular}

Clinical manifestation in relation to age group depicted that patients with age group 25-44 and 45-64 years were equally affected each accounting $32.5 \%$ of the cases followed by age group $15-24$ years accounting $21.3 \%$.Tinea unguium was found to be more in patients of age group 25- 44 years and tinea pedis in patients of age group 45- 64 years. Tinea capitis was common in patients of age group of 1-14 years (Table 2).

Table 2. Frequency of clinical manifestation in different age groups $(n=305)$.

\begin{tabular}{|c|c|c|c|c|c|c|}
\hline \multirow{2}{*}{ Site } & \multicolumn{5}{|l|}{ Age groups } & \multirow{2}{*}{ Total } \\
\hline & $1-14$ & $15-24$ & $25-44$ & $45-64$ & $>=65$ & \\
\hline Tinea capitis & 21 & 14 & 13 & 13 & 0 & $61(19.9 \%)$ \\
\hline Tinea corporis & 4 & 5 & 16 & 6 & 2 & $33(10.8 \%)$ \\
\hline Tinea cruris & 0 & 0 & 0 & 2 & 2 & $4(1.3 \%)$ \\
\hline Tinea unguium & 9 & 42 & 54 & 48 & 3 & $156(51.0 \%)$ \\
\hline Tinea pedis & 1 & 1 & 5 & 8 & 0 & $15(4.9 \%)$ \\
\hline Tinea faciei & 0 & 3 & 5 & 12 & 0 & $20(6.5 \%)$ \\
\hline Tinea manum & 0 & 0 & 6 & 10 & 0 & $16(5.2 \%$ \\
\hline Total & $35(11.5 \%)$ & $65(21.3 \%)$ & $99(32.5 \%)$ & $99(32.5 \%)$ & $7(2.3 \%)$ & $305(100 \%)$ \\
\hline
\end{tabular}

Of a total number of 305 patients suspected of dermatophytosis, fungi were detected and/ or isolated in 294 $(96.4 \%)$ patients. Fungal elements were detected in 265 $(86.9 \%)$ of clinical samples by $\mathrm{KOH}$ wet mount and 224 (73.4\%) clinical samples were culture positive. Clinical samples from 195 (63.9\%) were both culture and $\mathrm{KOH}$ positive. Among the study population fungi were neither detected nor showed visible fungal growth in culture in 11 (3.6\%) samples despite being obtained from lesions compatible to dermatophytosis (Table 3 ).

Table 3. Correlation of direct microscopy with culture $(n=305)$.

\begin{tabular}{lll}
\hline Test procedure & Number & Percentage \\
\hline KOH positive & 265 & 86.9 \\
Culture positive & 224 & 73.4 \\
KOH negative culture positive & 29 & 9.5 \\
KOH positive culture negative & 70 & 23.0 \\
Both KOH and culture positive & 195 & 63.9 \\
Both $\mathrm{KOH}$ and culture negative & 11 & 3.6 \\
\hline
\end{tabular}

As can be seen from table 4, of the total isolates, dermatophytes were the most common isolates accounting $130(58.0 \%)$ of the total isolates and this was followed by nondermatophyte molds that accounted $49(21.9 \%)$ of the total isolates. Yeasts were the least common isolates accounting 45 $(20.0 \%)$ of the total isolates. Further identification of the isolates showed the presence of ten species of dermatophytes of which $T$. violaceum was the dominant species accounting for $49(37.7 \%)$ dermatophyte isolates. This was followed by $T$. mentagrophytes and T. tonsuransthat accounted for 23 $(17.7 \%)$ and $22(17.0 \%)$, isolates of the dermatophyte respectively. $M$. nanum was the least common species of dermatophytes consisting of $1(0.8 \%)$ isolate of the dermatophyte. Candida albicans was the dominant yeast accounting $30(66.7 \%)$ of yeast isolates. The genus Aspergillus was the most common non-dermatophyte molds consisting of $13(26.5 \%)$ species and this was followed by
Alternaria species (8: 16.3\%) and Fusarium species (7: $14.3 \%)$.

Table 4. Mycological profile of the study $(n=224)$.

\begin{tabular}{|c|c|c|c|}
\hline Fungal category & Species & Number & Percentage \\
\hline \multirow{10}{*}{$\begin{array}{l}\text { Dermatophytes } \\
(\mathrm{n}=130,58 \%)\end{array}$} & T. violaceum & 49 & 37.7 \\
\hline & T. mentagrophytes & 23 & 17.7 \\
\hline & T. tonsurans & 22 & 17.0 \\
\hline & T. rubrum & 10 & 7.7 \\
\hline & T. soudanese & 5 & 3.8 \\
\hline & T. schoenleinii & 8 & 6.2 \\
\hline & T. verrucosum & 4 & 3.1 \\
\hline & M. audouiinii & 4 & 3.1 \\
\hline & M. nanum & 1 & 0.8 \\
\hline & E. flocosum & 4 & 3.1 \\
\hline \multirow[t]{5}{*}{ Yeasts $(n=45,20 \%)$} & C. albicans & 30 & 66.7 \\
\hline & C. krusei & 6 & 13.3 \\
\hline & C. glabrata & 5 & 11.1 \\
\hline & C. tropicalis & 4 & 8.9 \\
\hline & A. niger & 10 & 20.4 \\
\hline \multirow{10}{*}{$\begin{array}{l}\text { Non-dermatophytes } \\
(n=49,21.9 \%)\end{array}$} & Alternaria $s p$. & 8 & 16.3 \\
\hline & A. flavus & 1 & 2.0 \\
\hline & Cladosporium sp. & 7 & 14.3 \\
\hline & Fusarium sp. & 7 & 14.3 \\
\hline & Pencillium sp. & 3 & 6.1 \\
\hline & Scopulariopsis $s p$. & 3 & 6.1 \\
\hline & Curvularia sp. & 4 & 8.2 \\
\hline & Acremonium sp. & 2 & 4.1 \\
\hline & A.fumigatus & 2 & 4.1 \\
\hline & Syctalidium sp. & 2 & 4.1 \\
\hline
\end{tabular}

According to species frequency in different areas of involvement (tinea), T. violaceum was the most common pathogen in tinea unguium and tinea capitis, whereas $T$. mentagrophytes was the most common pathogen in tinea pedis and tinea manum. Seventy five percent of yeasts and $77.6 \%$ non dermatophyte molds were isolated from nails of which $C$. 
albicans and Aspergillus sp. were the major isolate among yeasts and non dermatophtye molds respectively (Table 5).

Table 5. Frequency of fungal isolates in relation to site of infection ( $n=224)$.

\begin{tabular}{|c|c|c|c|c|c|c|c|c|}
\hline \multirow{2}{*}{ Fungal isolates } & \multicolumn{7}{|c|}{ Clinical presentation } & \multirow{2}{*}{ Total } \\
\hline & T capitis & T corporis & T cruris & T unguium & $\mathbf{T}$ pedis & T faciei & T manum & \\
\hline T. violaceum & 17 & 4 & 0 & 19 & 1 & 6 & 2 & $49(21.9 \%)$ \\
\hline T. mentagrophytes & 5 & 1 & 1 & 7 & 3 & 1 & 5 & $23(10.3 \%)$ \\
\hline T. tonsurans & 7 & 4 & 0 & 9 & 0 & 1 & 0 & $21(9.4 \%)$ \\
\hline T. rubrum & 4 & 2 & 0 & 4 & 1 & 0 & 0 & $11(4.9 \%)$ \\
\hline T. schoenleinii & 3 & 3 & 0 & 2 & 0 & 0 & 0 & $8(3.6 \%)$ \\
\hline T. soudanese & 1 & 2 & 0 & 2 & 0 & 0 & 0 & $5(2.2 \%)$ \\
\hline M. audouinii & 1 & 0 & 0 & 3 & 0 & 0 & 0 & $4(1.8 \%)$ \\
\hline E. flocosum & 0 & 2 & 0 & 0 & 1 & 1 & 0 & $4(1.8 \%)$ \\
\hline M. nanum & 0 & 0 & 0 & 1 & 0 & 0 & 0 & $1(0.5 \%)$ \\
\hline A. niger & 0 & 1 & 1 & 8 & 0 & 0 & 0 & $10(4.5 \%)$ \\
\hline Alternaria sp. & 1 & 1 & 0 & 5 & 1 & 0 & 0 & $8(3.6 \%)$ \\
\hline Cladosporuim sp. & 2 & 2 & 0 & 3 & 0 & 0 & 0 & $7(3.1 \%)$ \\
\hline Fusarium sp. & 0 & 1 & 0 & 6 & 0 & 0 & 0 & $7(3.1 \%)$ \\
\hline Pencillium sp. & 0 & 0 & 0 & 3 & 0 & 0 & 0 & $3(1.3 \%)$ \\
\hline A. fumigatus & 0 & 0 & 0 & 2 & 0 & 0 & 0 & $2(0.9 \%)$ \\
\hline Acremonium sp. & 0 & 0 & 0 & 2 & 0 & 0 & 0 & $2(0.9 \%)$ \\
\hline Syctalidium sp. & 0 & 0 & 0 & 2 & 0 & 0 & 0 & $2(0.9 \%)$ \\
\hline A. flavus & 0 & 0 & 0 & 1 & 0 & 0 & 0 & $1(0.5 \%)$ \\
\hline C. albicans & 0 & 5 & 2 & 18 & 5 & 0 & 0 & $30(13.4 \%)$ \\
\hline C. krusei & 0 & 0 & 0 & 5 & 1 & 0 & 0 & $6(2.2 \%)$ \\
\hline C. glabrata & 0 & 0 & 0 & 4 & 1 & 0 & 0 & $5(2.2 \%)$ \\
\hline C. tropicalis & 0 & 0 & 0 & 3 & 1 & 0 & 0 & $4(1.8 \%)$ \\
\hline Total & $41(18.3 \%)$ & $30(13.4 \%)$ & $4(1.8 \%)$ & $118(52.7 \%)$ & $15(6.7 \%)$ & $9(4 \%)$ & $7(3.1 \%)$ & $224(100 \%)$ \\
\hline
\end{tabular}

Note $\mathrm{T}=$ tinea

\section{Discussion}

An accurate diagnosis of dermatophytosis is important for its successful treatment. The cost and long duration of the therapy, the risk of developing adverse drug reactions, and possible interactions with concomitant medications all underline the importance of accurate diagnosis of the condition before commencing therapy. Accurate diagnosis based on the clinical symptoms alone is often difficult. Currently, the diagnosis of dermatophytosis is confirmed by clinical examination and screening of the collected clinical specimen by direct microscopy $(\mathrm{KOH})$ and fungal culture. In various studies, $\mathrm{KOH}$ positivity rate varied from $35.6 \%$ to $100 \%$ and culture positivity rate varied from $36 \%$ to $66.7 \%[19,20]$. In these studies, the proportion of $\mathrm{KOH}$ negative isolates turning positive on culture varied widely from $5.6 \%$ to $56.7 \%$ [19]. Though $\mathrm{KOH}$ positivity rate $(86.9 \%)$ and $\mathrm{KOH}$ negative-culture positive fraction $(9.5 \%)$ in the present study were well within the reported range, a comparatively high culture positive rate $(73.4 \%)$ was achieved.

Dermtophytosis has been a common contagious disease and remain an important public health problem among people worldwide and particularly in developing countries. This is evident by the present study in which the prevalence of derdermatophyte and non dermatophyte fungi were 130 $(42.6 \%)$ and $94(30.8 \%)$ respectively with the overall prevalence of $73.4 \%$. A prevalence of $53 \%$ dermatophyte and $38 \%$ non dermatopyte fungi have been reported in a similar study conducted in Nigeria in 2012 by Ndako et al. [21] which iscomparably slightly higher than our finding. Another similar study conducted in Nigeria by Adefemi in 2013 [5] revealed that a prevalence of $5 \%$ dermatophyte and $15.4 \%$ non-dermatophyte fungi that is comparatively lower than our finding and aprevalence of $91 \%$ and $20.4 \%$ dermatophyte and non- dermatophyte fungi was documentedas an overall prevalence by the above two studies. Similarly, an overall prevalence of dermatophyte and non dermatophyte fungi of $66.7 \%$ and $71 \%$ have been depicted in similar studies conducted in India by Kannan et al [20] and in Saudi Arabia by Al Sheikh [6] respectively. Disparity in the prevalence of dermatophytosis in different studies could be resulted from differences in the lifestyle, socioeconomic conditions, risk factors associated with study subjects and environmental factors of study area $[1,3]$.

In terms of etiologic agents, of the total number 224 fungal isolates 130 were dermatophytes of which $72.3 \%$ was accounted by $T$. violaceum, T. mentagrophytes and $T$. tonsurans. Among the three dominant species, T. violaceum accounted $37.7 \%$ of the total isolates and our finding was compatible to studies conducted in Ethiopia [14-17], several other African [22- 23] and Asian countries [24, 25]. T. violaceum has been reported as one of the endemic dermatophyte in the horn of Africa and Asia by Ameen [3].

According to species frequency in different areas of involvement, $T$. violaceum, $T$. tonsurans and $T$. mentagrophytes were the most common dermatophytes recovered from tinea unguium and tinea capitis. Our finding in 
this regard is more or less similar with the findings of studies conducted in Ethiopia [16] India [20], Kenya [26] and USA [27]. Similarly T. mentagrophytes was the common dermatophyte isolated from tinea pedis and, tinea manum which is in line with other studies [28, 29].

Non dermatophytic molds (NDM) were isolated from 49 cases (21.9\%) mainly from nails (40 cases) with Aspergillus $s p$.as a major isolate accounting $26.6 \%$ of the total non dermatophyte mold isolates. Similarly, yeasts were isolated from 45 cases $(20.0 \%)$ mainly from nails (30 cases) with $C$. albicans as a major isolate accounting $66.7 \%$ of the total yeast isolates. A prevalence of $5 \%$ to $47.4 \%$ of non dermatophyte fungi from nails have been reported by various studies $[8,9$, $30,31]$ and a prevalence of $79.2 \%$ of $C$. albicans from a nail has been reported by Satpathi et al [9]. Though the prevalence of non dermatophyte molds and yeasts in the present study was well in reported ranges, a comparatively high prevalence rate of both non dermatophyte $(21.9 \%)$ molds and yeasts $(20 \%)$ was achieved. It is not known whether non dermatophyte fungi occur as true pathogen to the body of a health individual or exist as secondary invader in already damaged tissues and cause secondary tissue destruction. However, the non-dermatophyte fungi may be considered as important pathogen with a high index of suspicion in evaluating the patients with culture negative for dermatophytes or those subjects ending up in treatment failure. Isolation and characterization of non dermatopyte fungi in patients suspected of dermatophytosis is the first report to the country.

The present study showed that more females were affected by dermatophytes than males, female male ratio being 2.2:1. Earlier studies also indicated a higher prevalence of dermatophytes in females compared to males [32-35]. Meanwhile some other earlier studies recorded a higher prevalence of dermatophytes in males than females $[5,36]$.

The dominant type of clinical manifestation of dermatophytosis varies considerably in different studies reported in literature. In a study conducted in India, tinea corporis $(35.4 \%)$ was the predominant clinical condition followed by tinea cruris (16.8\%) and tinea capitis (16.7\%) [32). Similar study conducted in Iran between March 2005 and March 2007 by Rassai et al. [33] revealed that tinea cruris and tinea corporis were the most common clinical manifestation. A 7 years (1997-2003) survey of dermatophytosis in Crete, Greece conducted by Maraki et al [34] revealed that tinea unguium was the predominant clinical manifestation. A study carried out by Devliotou-Panagiotidou et al. [35] between 1981 and 1990 in Greece depicted that tinea pedis was the most frequent clinical manifestation. Adefemi et al. [5] reported tinea capitis as a predominant clinical manifestation.In our study, tinea unguium was the dominant clinical manifestation involving $51.1 \%$ of the total cases of dermatophytosis, similar to many other reports [34, 36]. Tinea capitis was the second clinical manifestation accounting $61(20 \%)$ of dermatophytosis as has been observed in other studies [5, 26, 37]. Tinea corporis was the third common clinical presentation accounting 33 (10.8\%) and this clinical manifestation has been reported as a dominant clinical manifestation by earlier similar studies $[23,32$, and 33].

In the present study persons of all age groups were susceptible to dermatophytosis but it appeared to be more common in adults of age group 25-44 and 45-64 years each accounting $32.5 \%$ of the cases as they are physically active outdoors. Our finding in this regard was compatible with the findings of others $[14,16]$. As universally reported by most of the workers, tinia capitis is an infection of childhood. In the present study a total 61 patients with tinea capitis, 21 patients were in age group of 1-14. Similar results were reported by earlier researches $[6,38]$. The changing pattern of hormones after puberty [39] and production of inadequate amounts of inhibitory fatty acids before puberty [40] are responsible for a decrease of tinea capitis with age. On the other hand, tinea unguium was more frequent in the elderly population with an age group of 25-64. Reduced growth rate of the ungual plate, an increase in trauma rates, poor peripheral circulation and inability to maintain good foot care could attributed to this [41]. On the other hand tinea pedis was a dominant clinical manifestation in age group 45-64 years which was in agreement with the findings of Lange et al and Caputo et al. $[42,43]$. This study has also revealed that $T$. violaceum, $T$. mentagropphytes and $T$. tonsurans were the commonly isolated dermatophytes in different age categories.

\section{References}

[1] Havlickova B, Czaika VA, Friedrich M. Epidemiological trends in skin mycoses worldwide. Mycoses 2008; 51:2-15.

[2] Popoola SOT, OJO AD, Alabi OR. Prevalence of dermatophytosis in junior secondary school children in Ogun State, Nigeria. Mycoses 2006; 49: 499-503.

[3] Ameen M. Epidemiology of superficial fungal infections. Clin. Dermatol. 2010; 28:197-201.

[4] Nweze EI. Dermatophytosis among children of Fulani/Hausa living in South Eastern Nigeria. Revistalberoamericana de Micol. 2010: 27: 191-194.

[5] Adefemi SA, Odeigah LO, Alabi KM. Prevalence of dermatophytosis among primary school children in Oke-oyi community of Kwara state. Nigerian J. Clin. Practice 2011; 14: 23-28.

[6] Al Shekh H. Epidemiology of dermatophtes in the eastern province of Saudi Arabia. Res. J. Micobiol 2009; 4: 229-234.

[7] Erbagci Z, Tuncel AA, Yasemin ZY, Balci I. A prospective epidemiologic survey on the prevalence of onychomycosis and dermatophytosis in male boarding school residents Mycopathologia 2005; 159: 347-35.

[8] Beena S, Sreeja. MV, Bhavana PR, SreenivasaBabu S. Onychomycosis: prevalence and its etiology in a tertiary care hospital, south India. Int. J. Health Sci. Res 2013; 3::81-85.

[9] Satpathi P, Achar A, Banerjee D, Maiti A,Sengupta M, Mohata A. Onychomycosis in EasternIndia - study in a peripheral tertiary care centre. J. Pakistan Assoc. Dermatol 2013; 23:14-19. 
[10] Asadi MA, Dehghan R, Sharif. MR.Epidemiologic study of onychomycosis and tinea pedis in Kashan, Iran. Jundishapur J. Microbiol 2009; 2: 61-64.

[11] Bramono K, Budjmlja U. Epidiomology of onychomycosis in Indonesia: Data obtained from three Indonian studies. Jpn. J. Mycol 2005; 46: 171-176.

[12] Hay RJ. Fungal infections. In: Warrell DA, Cox TM, Firth JD and Benz EJ Jr. (eds). Oxford Textbook of Medicine, ed 4. Oxford University Press, Oxford, 2003; P. 7.12.1.

[13] Ilkit M. Favus of the scalp: an overview and update. Mycopathologia 2010; 170: 143-154.

[14] Figueroa JI, Hawranek T, Abraha A, Hay RJ. Tinea capitis in Southwestern Ethiopia: a study of risk factors for infection and carriage. Int. J. Dermatol 1997; 36: 661-6.

[15] Figueroa JL, Hawranek T, Abraha A, Hay RJ. Dermatology in Southwestern Ethiopia: rationale for a community approach. Int. J. Dermatol 1998; 37: 752-8.

[16] Woldeamanuel Y. Mengistu Y, Chryssanthou E, Petrini B. Dermatophytosis in Tulugudu Island, Ethiopia, Med. Mycol. $2005 ; 43 ; 79-82$.

[17] Woldeamanuel Y, Leekassa R, Chryssanthou E, Mengistu Y, Petrini B. Prevalence of Tinea capitis in Ethiopoan school children. Mycoses 2005; 45: 137-41.

[18] Kern M. Medical mycology, a self instructional text. Philadelphia, F. D Davis Company, 2nd ed. 1985.

[19] Mohanty JC, Mohanty SK, Sahoo RC et al. Diagnosis of superficial mycoses by direct microscopy - a statistical evaluation. IJDV, 1999; 65: 72 - 4 .

[20] Kannan P, Janaki C, Selvi GS. Prevalence of dermatophytes and other fungal agents isolated from clinical specimens. Indian J Med. Microbiol 2006; 24 (3):212-215.

[21] Ndako JA, Osemwegie OO, Spencer THI, Olopade BK, Yunusa GA, Banda J. Prevalence of Dermatophytes and other associated Fungi among school children. Global Advanced Res. J. Medicine and Medical Sci 2012; 1(3): 049-056.

[22] Gangoom AM, Elyazachi MB, Al-Ani SM, Duwb GA. Tinea capitis in Benghazi, Libya. Int. J.Dermatol 2000; 39: 263-5.

[23] Ellabib MS, Agaj M, Khalifa Z, Kavanagh K. Trichophyton violaceum is the dominant cause of tinea capitis in children in Tripoli, Libya: results from a two-year survey. Mycopathologia 2002; 153: 145-7.

[24] Ali-Shtayeh MS, Salameh AA, Abu-Ghdeib SI, Jamous RM, Khrahir H. Prevalence of tinea capitis as well as asymptomatic carriers in school children in Nablus area (Palestine). Mycoses 2002; 45: 188-94.

[25] Hussain I, Aman S, Haroon TS, Jahangir M, Nagi AH. Tinea capitis in Lahore, Pakistan. Int.J.Dermatol 1994; 33: 255-7.

[26] Chepchirchir A, BiiC,Ndinya -Achola JO.Dermatophyte infections in primary school children in Kibera Slums of Nairobi. East African Medical J. 2009; 86: 60-68.

[27] Shelley S. M, Liliana M, Andrew S, Bernard C, William G. M.
Isolation of Trichophyton violaceum and Trichophyton soudanense in Baltimore, Maryland. J. Clin. Microbiol. 2007; 45:461.

[28] Shahla BN, Effat K, Mehdi AA. Study Of Dermatophytosis Infections In Dermatology Clinic Of Sina Hospital - Tabriz. Ege T1p Dergisi, 2007; 46: 21 - 25.

[29] Meritxell PG, Josep MT, Antoni M, Sonia S, Gemma G, Laura T, Marta P. Prevalence of tinea pedis, tinea unguium of toenails and tinea capitis in school Children from Barcelona. Rev Iberoam Micol 2009; 26:228-232.

[30] Reddy KN, Srikanth BA, Ram Sharan TR, Biradar PM. American J. Dermatol. And Venereol 2012; 1: 35-40.

[31] Lone R., Showkat HR, Bashir D, Khursheed S, Hussain A, Sarmast RH. ClinicoMycological Pattern of Onychomycosis. Eur J. Gen. Med 2013; 10:150-153.

[32] Balakumar S, Rajan S, Thirunalasundari T, Solomon Jeeva S. Epidemiology of dermatophytosis in and around Tiruchirapalli, Tamilnadu, India. Asian Pac. J. Trop. Dis 2012; 2: 286-289.

[33] Rassai1 S, Feily A, Sina N, Derakhshanmehr F. Some Epidemiological Aspects of Dermatophyte Infections in Southwest Iran. Acta Dermato venerol Croat.2011; 19:13-15.

[34] Maraki S, Nioti E, Mantadakis E, Tselentis Y.A 7-year survey of dermatophytoses in Crete, Greece. Mycoses 2007; 50: 481-484.

[35] Devliotou-Panagiotidou D, Koussidou-Eremondi T, Badillet G. Dermatophytosis in North Greece during the decade 1981-1990. Mycoses 1995; 38: 151- 157.

[36] Vena GA, Chieco P, Posa F,Garofalo A, Bosco A, Cassano N. Epidemiology of dermatophytoses: retrospective analysis from 2005 - 2010 and comparison with previous data from 1975. New Microbilogica. 2012; 35: 207-213.

[37] Wahab AS, Al-Fouzan, Nanda A, kubec K. Dermatophytosis of children in Kuwait: A prospective study. Int. J. Dermatol.1993; 32: $798-801$.

[38] Attapattu MC. A study of tinea capitis in Sri-lanka. J. Medical and Veterinary Mycol 1989; 27: 27-32.

[39] Oliveira JAA, Barros JA, Cortez ACA, Oliveira JSRL. Superficial mycoses in the city of Manaus. An. Bras Dermatol $2006 ; 238-243$.

[40] David G, Richard C.B et al. Medical Microbiology: a guide to microbial infections, pathogenesis, immunity, laboratory diagnosis and control. Churchill Livingstone. Ed 16. 2003; 568-579.

[41] Kaur R, Kashyap B, Bhalla P. Onychomycosis - Epidemiology, diagnosis and management. IJMM 2008; 26: 108 - 16.

[42] Lange M, Nowicki R, Baranska- RybakW, Bykowska B. Dermatophytosis in children and adolescents in Ghansk, Poland. Mycoses 2003; 47: 326-329.

[43] Caputo R, De Boulle K, Del Rosso J, Nowicki R. Prevalence of superficial fungal infections among sports-active individuals: results from the Achilles survey, a review of the literature. J. Eur Acad. Dermatol. Venereol 2001; 15:312-6. 